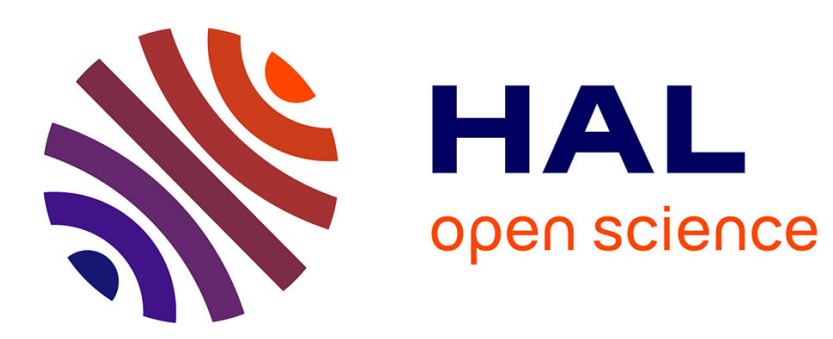

\title{
Etude de silicium implanté à l'arsenic par effet de transport. Influence du recuit thermique
}

\author{
C. Christofidès, G. Ghibaudo, H. Jaouen
}

\section{To cite this version:}

C. Christofidès, G. Ghibaudo, H. Jaouen. Etude de silicium implanté à l'arsenic par effet de transport. Influence du recuit thermique. Revue de Physique Appliquée, 1987, 22 (6), pp.407-412. 10.1051/rphysap:01987002206040700 . jpa-00245555

\section{HAL Id: jpa-00245555 https://hal.science/jpa-00245555}

Submitted on 1 Jan 1987

HAL is a multi-disciplinary open access archive for the deposit and dissemination of scientific research documents, whether they are published or not. The documents may come from teaching and research institutions in France or abroad, or from public or private research centers.
L'archive ouverte pluridisciplinaire HAL, est destinée au dépôt et à la diffusion de documents scientifiques de niveau recherche, publiés ou non, émanant des établissements d'enseignement et de recherche français ou étrangers, des laboratoires publics ou privés. 
Classification

Physics Abstracts

$61.70 \mathrm{~T}-61.80 \mathrm{~J}-72.20 \mathrm{D}-81.40$

\title{
Etude de silicium implanté à l'arsenic par effet de transport. Influence du recuit thermique
}

\author{
C. Christofidès, G. Ghibaudo et H. Jaouen \\ Laboratoire de Physique des Composants à Semiconducteurs, ENSER-INPG, 23, av. des Martyrs, \\ 38031 Grenoble, France
}

(Reçu le 17 janvier 1986, révisé le 9 septembre, accepté le 12 février 1987)

\begin{abstract}
Résumé. - Une étude des effets du recuit thermique sur les propriétés de transport dans le silicium implanté à l'arsenic est présentée. En particulier, la résistance carré et la mobilité de Hall obtenues par la méthode de Van der Pauw ont été mesurées en fonction de la température $(77 \mathrm{~K}$ à $300 \mathrm{~K}$ ) sur des échantillons $\mathrm{N}^{+} / \mathrm{N}$ non recuits et soumis à des recuits isothermes entre $300^{\circ} \mathrm{C}$ et $1100^{\circ} \mathrm{C}$. L'énergie d'activation du processus de guérison des dommages causés par l'implantation ionique, trouvée de l'ordre de $0,65 \mathrm{eV}$, est attribuable à une restructuration locale des couches. Au voisinage de $420^{\circ} \mathrm{C}$, la résistance carré varie de plusieurs ordres de grandeurs et les variations de la mobilité de Hall avec la température traduisent un changement notable des mécanismes de collisions avec la température de recuit.
\end{abstract}

Abstract. - A study of the effects of thermal annealing on the transport properties in arsenic implanted silicon films is reported. In particular, the sheet resistance and the Hall mobility obtained by the Van der Pauw method is measured as a function of temperature $(77 \mathrm{~K}$ to $300 \mathrm{~K})$ on $\mathrm{N}^{+} / \mathrm{N}$ non annealed and isothermally annealed samples between $300^{\circ} \mathrm{C}$ to $1100^{\circ} \mathrm{C}$. The activation energy of the recovery process of the ionic implantation damages found on the order to $0.65 \mathrm{eV}$ is likely attributed to a local reconstruction of the layer. Around $420^{\circ} \mathrm{C}$, the sheet resistance varies on several orders of magnitude and the Hall mobility variations with temperature clearly put toward a noticeable change of the scattering processes with annealing temperature.

\section{Introduction.}

Il est bien connu que l'implantation ionique des impuretés dans le silicium provoque une forte détérioration du matériau (amorphisation) ou création de régions fortement désordonnées [1].

Ces effets ont été très largement étudiés par diverses méthodes telles que :

- physico-chimiques (R.B.S. [2, 3], TEM [4]) ;

- optiques (ellipsométrie, spectroscopie et absorption infrarouge $[5,6]$.

Des méthodes électriques telles que la D.L.T.S. $[7,8,9]$ ou celle des quatre pointes ont été également utilisées [10].

Cependant peu d'études ont été consacrées à l'examen des propriétés de transports (conductivité, effet Hall) dans de telles couches fortement désordonnées par implantation ionique.

L'objectif de ce travail est d'analyser les principaux mécanismes de restructuration des couches endommagées par implantation par le biais des effets de transport dans le silicium implanté à l'arsenic, d'une part en fonction de la température de mesure $(77 \mathrm{~K}$ à $300 \mathrm{~K})$ et d'autre part, en fonction de la température de recuit (300 à $1100^{\circ} \mathrm{C}$ ).

\section{Détails expérimentaux.}

2.1 CaRACTÉRISTIQUeS DES ÉCHANTILlons. Les échantillons ont été réalisés sur des substrats de silicium (100) de type $\mathrm{N}\left(10^{15} \mathrm{~cm}^{-3}\right)$ implanté à la température ambiante à l'arsenic avec une dose $8 \times 10^{14} \mathrm{~cm}^{-2}$ et une énergie d'implantation de $120 \mathrm{keV}$. Une partie de ces échantillons a été recuite à différentes températures $\left(300{ }^{\circ} \mathrm{C}<T_{\mathrm{a}}<1100^{\circ} \mathrm{C}\right)$ pendant une heure dans des fours thermiques.

Les échantillons de silicium de $4 \mathrm{~mm}$ de côté ont été montés sur des plaquettes de céramique. Quatre contacts ohmiques ont été réalisés en surface par évaporation d'aluminium puis soudure à ultrason. 


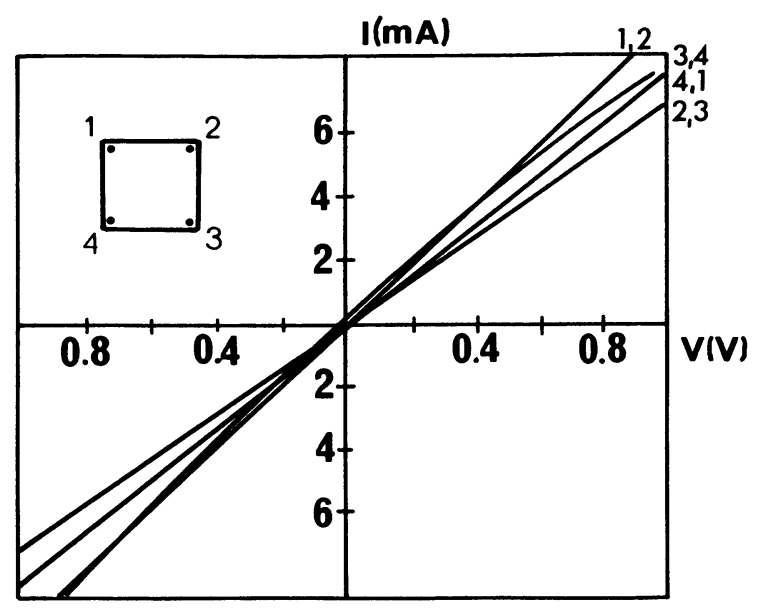

Fig. 1. - Caractéristiques $I(V)$ typiques pour des échantillons de silicium implantés munis de contacts obtenus par évaporation d'aluminium.

[Typical $I(V)$ characteristics for the implanted silicon samples with evaporated aluminum contacts.]

La figure 1 donne un exemple de caractéristiques $I(V)$ obtenues sur de tels échantillons.

2.2 Méthodes DE MeSure. - Les coefficients de transport de ces échantillons ont été mesurés par la méthode de Van der Pauw [11].

La tension de Hall $V_{\mathrm{H}}$ est déterminée par la relation :

$$
V_{\mathrm{H}}=\frac{V(+B)-V(-B)}{2}
$$

où $V$ est la tension entre 2 contacts croisés et $B$ l'induction magnétique $(B=0,6$ tesla dans ce travail).

La résistance carré $R_{\mathrm{s}}$ est alors donnée par:

$$
R_{\mathrm{s}}=\frac{\pi f}{2 \ln 2}\left(R_{1}+R_{2}\right)
$$

où $f$ est la fonction de Van der Pauw, $R_{1}$ et $R_{2}$ sont les résistances entre deux contacts successifs [11].

La mobilité de Hall $\mu_{H}$ s'obtient par:

$$
\mu_{\mathrm{H}}=\frac{V_{\mathrm{H}}}{I B R_{\mathrm{S}}}
$$

où $I$ est le courant injecté entre deux contacts non successifs (de l'ordre de $10^{-6} \mathrm{~A}$ dans notre cas).

\section{Résultats expérimentaux.}

Les figures 2 et 3 représentent respectivement les variations de la résistance carré et de la mobilité de Hall (mesurée à $300 \mathrm{~K}$ ) en fonction de l'inverse de la température de recuit $T_{\mathrm{a}}$.

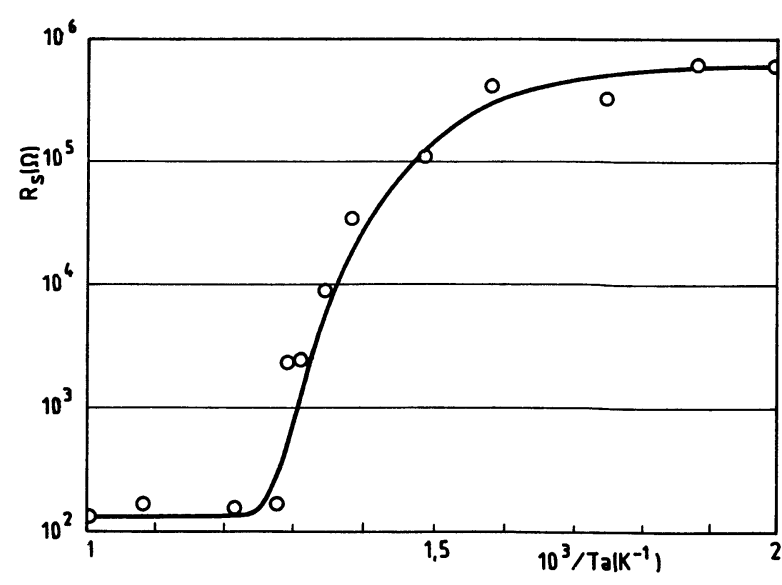

Fig. 2. - Variation de la résistance carré mesurée à $300 \mathrm{~K}$ en fonction de l'inverse de la température de recuit (points expérimentaux et courbe théorique obtenue avec la relation (4a)).

[Variation of the sheet resistance measured at $300 \mathrm{~K}$ as a function of the inverse of the annealing temperature (experimental points and theoretical curve obtained by relation $(4 \mathrm{a}))$.]

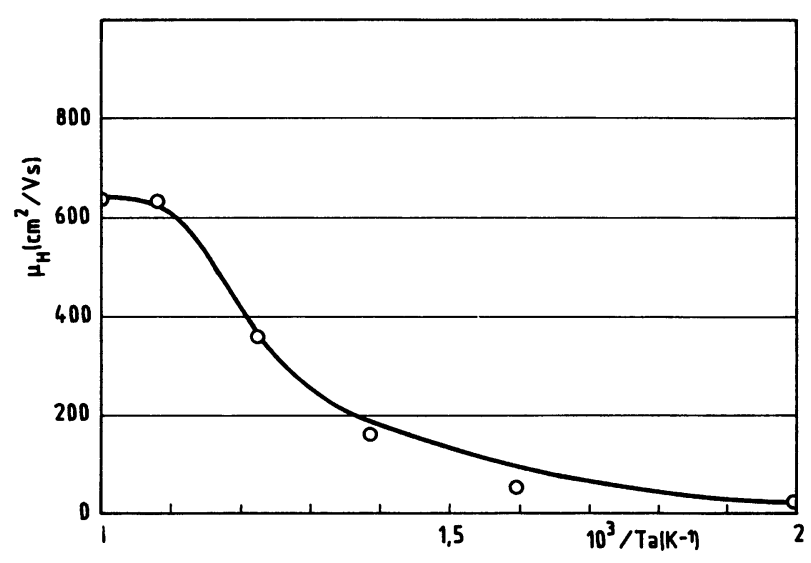

Fig. 3. - Variation de la mobilité de Hall mesurée à $300 \mathrm{~K}$ en fonction de l'inverse de la température de recuit (points expérimentaux et courbe théorique obtenue avec la relation (4b)).

[Variation of the Hall mobility measured at $300 \mathrm{~K}$ as a function of the inverse of the annealing temperature (experimental points and theoretical curve obtained by relation (4b)).]

La température de recuit à partir de laquelle la résistance carré $R_{\mathrm{s}}$ diminue fortement est de l'ordre de $420^{\circ} \mathrm{C}$; autour de cette température $R_{\mathrm{s}}$ varie de $5,50 \times 10^{5} \Omega$ à $100 \Omega$ et $\mu_{H}$ varie de 16 à $670 \mathrm{~cm}^{2} / \mathrm{Vs}$.

La figure 4 montre les variations des résistances carré $R_{\mathrm{s}}$ en fonction de la température de mesure avec comme paramètre la température de recuit $T_{\mathrm{a}}$. On constate que $R_{\mathrm{s}}$ varie faiblement avec la 


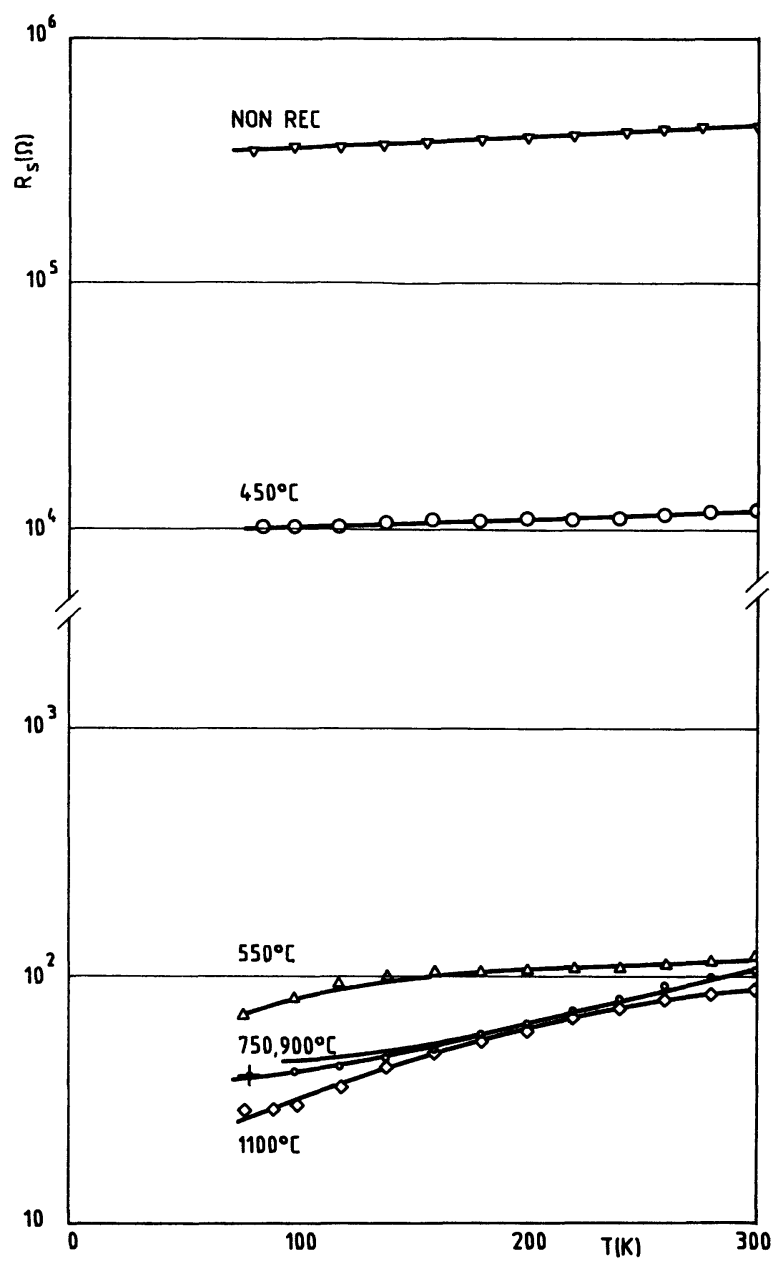

Fig. 4. - Variation de la résistance carré en fonction de la température de mesure pour des échantillons recuits à différentes températures.

[Variation of the sheet resistance as a function of temperature for samples annealed at different temperatures.]

température entre $77 \mathrm{~K}$ et $300 \mathrm{~K}$. Pour les échantillons non et faiblement recuits la dépendance en température est moins prononcée.

La figure 5 présente les variations de la mobilité de Hall $\mu_{H}$ en fonction de la température de mesure pour différentes conditions de recuit. On note principalement une forte atténuation de la dépendance de $\mu_{H}$ en température pour des échantillons faiblement recuits.

\section{Discussion des résultats.}

4.1 EFFET DE LA TEMPÉRATURE DE RECUIT $T_{\mathrm{a}}$. La figure 2 qui représente $R_{\mathrm{s}}$ en fonction de $1 / T_{\mathrm{a}}$ met en évidence le changement de structure dans les échantillons implantés intervenant lors du recuit thermique. Compte tenu de l'énergie et de la dose d'implantation, la couche en surface de l'ordre de $300 \mathrm{~nm}$ d'épaisseur n'est pas entièrement amorphe mais présente en surface une couche amorphisée

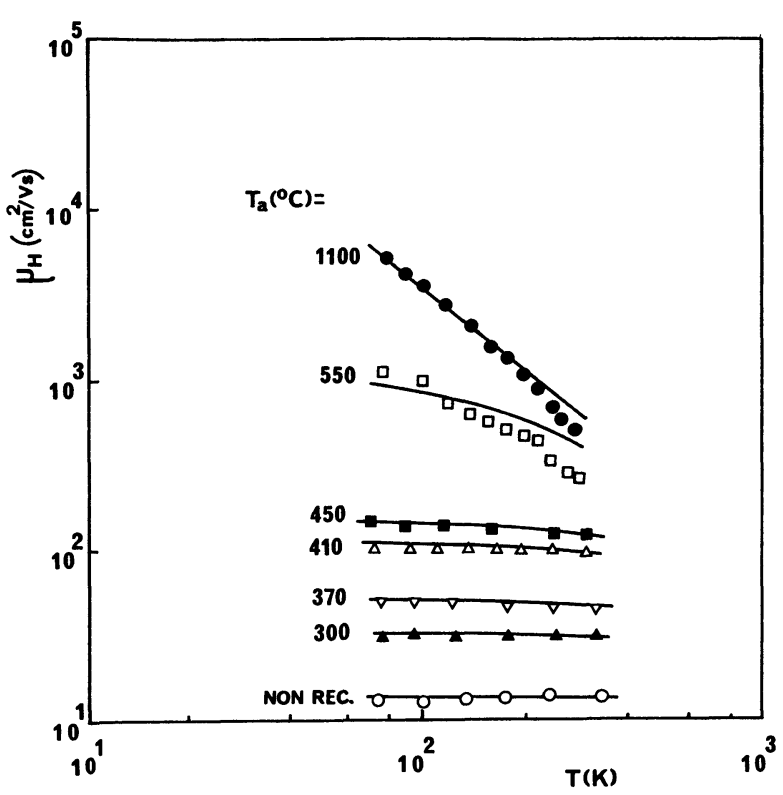

Fig. 5. - Variation de la mobilité de Hall $\mu_{H}$ en fonction de la température de mesure pour des échantillons recuits à différentes températures (points expérimentaux et courbes théoriques obtenues avec la relation (7)).

[Variation of the mobility $\mu_{H}$ as a function of temperature for samples annealed at different temperatures (experimental points and theoretical curves obtained by relation (7)).]

derrière laquelle existe une région fortement endommagée dans laquelle des zones amorphes sont entourées par des régions à fort taux de défauts $[10,12]$. La température de transition de $420^{\circ} \mathrm{C}$ correspond d'après Nelson [13] à une restructuration d'une couche comportant des «clusters » amorphes d'un diamètre de l'ordre de $4 \mathrm{~nm}$.

Dans ce type de couches non totalement amorphisées le mécanisme de guérison classique par épitaxie en phase solide à partir du substrat ne semble pas convenir vu la faible valeur de la température de transition. Une analyse de ces résultats peut être faite selon un mécanisme de restructuration locale des couches décrit par une cinétique globale de la forme [14] :

$$
\begin{gathered}
R_{\mathrm{s}}=\left(R_{\mathrm{si}}-R_{\mathrm{sf}}\right) \exp (-t / \tau)+R_{\mathrm{sf}} \\
\mu_{\mathrm{H}}=\left(\mu_{\mathrm{Hi}}-\mu_{\mathrm{Hf}}\right) \exp (-t / \tau)+\mu_{\mathrm{Hf}}
\end{gathered}
$$

où

$R_{\mathrm{si}}, \mu_{\mathrm{Hi}} \quad$ : résistance carré et mobilité d'un échantillon non recuit,

$R_{\mathrm{sf}}, \mu_{\mathrm{Hf}}$ : résistance carré et mobilité d'un échantillon recuit à haute température $\left(1100{ }^{\circ} \mathrm{C}\right)$,

: durée du recuit isochrone (dans notre cas $t$ est égal à une heure),

: temps de relaxation du processus. 
Le tracé du logarithme de la résistance carré réduite $Q$, définie par

$$
Q=\frac{R_{\mathrm{si}}-R_{\mathrm{sf}}}{R_{\mathrm{s}}-R_{\mathrm{sf}}}
$$

dans un plan semilogarithmique en fonction de $1 / T_{\mathrm{a}}$ (cf. Fig. 6) montre que le temps de relaxation est activé thermiquement. La pente caractéristique est proportionnelle à l'énergie d'activation $E_{\mathrm{a}}$ du temps de relaxation $\tau$ qui peut s'écrire sous la forme :

$$
\tau=\tau_{0} \exp \left(\frac{E_{\mathrm{a}}}{k T}\right)
$$

où $k$ est la constante de Boltzmann.

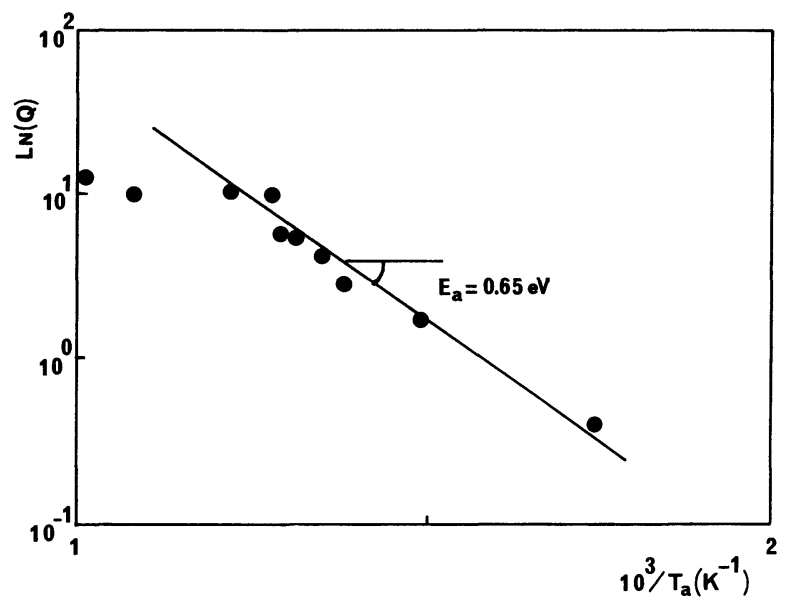

Fig. 6. - Variation de $\operatorname{Ln}(Q)$ avec l'inverse de la température de recuit $T_{\mathrm{a}}$ : détermination de l'énergie d'activation du temps de relaxation.

[ $\operatorname{Ln}(Q)$ variation with reverse annealing temperature $T_{\mathrm{a}}$ : determination of the activation energy of the relaxation time.]

Sur la figure 2 est représentée la courbe théorique (en continu) obtenue grâce aux relations (4) et (6) avec pour paramètres $E_{\mathrm{a}}=0,65 \mathrm{eV}$ et $\tau_{0}=$ $3,6 \times 10^{-6} \mathrm{~h}$ et qui est en bon accord avec les points expérimentaux.

Dans ce processus de guérison de la couche à faible énergie d'activation, la recristallisation d'une phase amorphe par épitaxie en phase solide dont l'énergie d'activation est de 2 à $3 \mathrm{eV}$ [15], ne semble pas dominant. Un réarrangement local de groupes de défauts ponctuels résultant des dommages causés par l'implantation, qui sont dans ce cas vraisemblablement associés à la formation de silicium et d'arsenic interstitiels ainsi que de lacunes [1], semble davantage constituer le mécanisme prépondérant de guérison des couches.
En effet, l'énergie d'activation de $0,65 \mathrm{eV}$ correspond dans ce cas à une énergie de réarrangement par migration des défauts ponctuels. Il semble que, vu les énergies de migration du silicium interstitiel de l'ordre de 0,2 à $0,5 \mathrm{eV}$ [16], ces derniers soient responsables pour l'essentiel de ce mécanisme de restructuration locale.

En ce qui concerne l'évolution de la mobilité de Hall avec la température de recuit, conforme par ailleurs à la relation (4b), on notera (Fig. 3) que la variation de son amplitude est beaucoup plus faible (de l'ordre de 40) que celle de la résistance carré qui est de l'ordre de $5,5 \times 10^{3}$. Cela montre que dans l'évolution de $R_{\mathrm{s}}$, intervient non seulement le facteur dû à la mobilité mais surtout l'accroissement de la concentration des porteurs consécutif à l'activation électrique des impuretés implantées (arsenic). De plus l'évolution de $\mu_{\mathrm{H}}$ avec la température de recuit met clairement en évidence la diminution après recuit du taux de collision.

4.2 EFFET DE LA TEMPÉRATURE DE MESURE. - Sur les figures 3 et 4 , on remarque que la variation des coefficients de transport est d'autant plus importante que la température de recuit $T_{\mathrm{a}}$ est élevée. Les valeurs de $R_{\mathrm{s}}$ et $\mu_{\mathrm{H}}$ obtenues après recuit à hautes températures sont tout à fait conformes à celles d'un silicium massif ayant un dopage moyen de $4-5 \times 10^{17} \mathrm{~cm}^{-3}$. Dans ce cas les variations de $\mu_{H}$ avec la température vérifient une loi usuelle en $T^{-3 / 2}$ caractéristique d'un mécanisme de collision sur le réseau [17]. La variation de $R_{\mathrm{s}}$ correspondante est alors en bon accord avec celle de $\mu_{H}$ [18].

En revanche, l'absence de dépendance en température de $\mu_{\mathrm{H}}$ et de $R_{\mathrm{s}}$ pour les échantillons non recuits souligne l'apparition d'un certain degré d'amorphisation des couches $[19,20]$. De plus, les valeurs des mobilités de Hall observées sont de l'ordre de $10 \mathrm{~cm}^{2} / \mathrm{Vs}$ ce qui montre à nouveau que les couches implantées que nous étudions ne sont pas entièrement amorphisées (cf. § 4.1) mais seulement fortement désordonnées. Cela suggère qu'un des mécanismes de collision prépondérant provient d'une forte densité de centres neutres [17].

Comme nous l'avons vu précédemment, les couches étudiées semblent être formées de clusters de $4 \mathrm{~nm}$ de diamètre ce qui nous permet de les considérer comme des centres neutres de collision compte tenu de leur inactivité électrique.

Dans ce matériau deux mécanismes de collision seront alors considérés :

1) l'un identique à celui que l'on a dans la couche recuite,

2) l'autre lié aux centres neutres que constituent les clusters amorphes.

En utilisant la règle de Mathiessen [17], la mobilité effective de la couche $\mu$ s'exprime généralement 
pour les semiconducteurs non dégénérés sous la forme :

$$
\frac{1}{\mu}=\frac{1}{\mu_{\mathrm{cris}}}+\frac{1}{\mu_{\mathrm{clus}}}
$$

où

$$
\mu_{\text {cris }}=580(T / 300)^{-1.5}\left(\mathrm{~cm}^{2} / \mathrm{Vs}\right) \text {, }
$$

représente la loi de mobilité dans la matrice de silicium exempte de centres neutres et,

$$
\mu_{\mathrm{clus}}=\frac{q^{3} m^{*}}{20 \hbar^{3} \varepsilon} \frac{1}{N}
$$

est la loi usuelle de mobilité pour des centres neutres de collision [17] avec :

$q$ : charge absolue de l'électron,

$m^{*}$ : masse effective de l'électron,

$\hbar$ : constante réduite de Planck,

$\varepsilon$ : permittivité du silicium,

$N$ : densité de clusters amorphes.

Il résulte de la composition de ces deux mécanismes, que la mobilité dépend de la température de mesure selon l'intensité relative des deux mécanismes. La densité des clusters $N$, obtenue par ajustement des courbes théoriques aux points expérimentaux (cf. Fig. 5), peut être déterminée approximativement en fonction de la température de recuit (cf. Tableau I) sachant que l'erreur introduite par l'application de la règle de Mathiessen n'excède pas 20$30 \%$ [21].

Tableau I. - Valeurs de la densité de clusters $N$ pour différentes températures de recuit.

[Values of the cluster density $N$ for different annealing temperatures.]

\begin{tabular}{cc}
\hline$T_{\mathrm{a}}\left({ }^{\circ} \mathrm{C}\right)$ & $N\left(\mathrm{~cm}^{-3}\right)$ \\
\hline Non Rec. & $6 \times 10^{20}$ \\
\hline 300 & $2,7 \times 10^{20}$ \\
\hline 370 & $1,7 \times 10^{20}$ \\
\hline 410 & $8 \times 10^{19}$ \\
\hline 450 & $6 \times 10^{19}$ \\
\hline 550 & $8 \times 10^{18}$ \\
\hline 1100 & 0 \\
\hline
\end{tabular}

La densité surfacique de clusters, de l'ordre de $7 \times 10^{13} / \mathrm{cm}^{2}$, conduit, compte tenu de la dose d'implantation, à estimer la taille des clusters à une dizaine d'atomes d'impuretés. On note de plus, d'après le tableau I, la forte diminution de la densité de clusters avec la température de recuit.

\section{Conclusion.}

Dans ce travail nous avons étudié les propriétés de transport dans les couches de silicium implanté à l'arsenic.

Les résultats principaux de cette étude sont :

1) la forte influence de la température du recuit sur la valeur des coefficients de transport (la résistance carré varie de plusieurs ordres de grandeurs entre 400 et $450^{\circ} \mathrm{C}$ ),

2) un changement notable du comportement des coefficients de transport, en particulier de la mobilité avec la température de mesure.

En ce qui concerne le processus de guérison des couches endommagées par implantation ionique, nous avons mis en évidence une énergie d'activation relativement faible $(0,65 \mathrm{eV})$ probablement caractéristique d'un mécanisme de réarrangement local par migration de silicium interstitiel.

Le changement du comportement de la mobilité de Hall peut être interprété de façon qualitative en considérant que le matériau implanté est constitué de régions amorphes de diamètres de l'ordre de 4 à $5 \mathrm{~nm}$ distribuées dans une matrice de silicium cristallin. D'après ce modèle simple, la densité de clusters amorphes a été estimée à $6 \times 10^{20} / \mathrm{cm}^{3}$ pour les couches non recuites.

Les résultats expérimentaux reportés ici mettent en évidence la situation particulière des semiconducteurs désordonnés. En effet, dans les semiconducteurs cristallins les propriétés de transport sont relativement bien interprétées par les théories classiques du transport. Les semiconducteurs amorphes font également l'objet de nombreux travaux théoriques qui permettent d'expliquer qualitativement les mécanismes de transport.

En revanche, pour les matériaux intermédiaires, la catégorie des matériaux inhomogènes, situés entre les matériaux complètement cristallins et les matériaux amorphes, les propriétés du transport sont difficilement interprétées soit par les modèles classiques des solides cristallins soit par les modèles relatifs aux matériaux amorphes.

Les couches de silicium implanté non recuites ou insuffisamment recuites sont un exemple typique de ces matériaux inhomogènes pour lesquels un effort de modélisation de transport devient nécessaire. 


\section{Bibliographie}

[1] Gibbons, J. F., Proc. IEEE 609 (1972) 1062.

[2] Picraux, S. T., Defects in semiconductors, edited by J. Narayan and T. Y. Tan (North Holland-New York) 2 (1981) 135.

[3] Paulson, W. M. and Corbett, J. W. (North Holland-New York) 14 (1983) 523.

[4] Krakow, W., TaN, T. Y. and Foell, H., Defect in semiconductors, edited by J. Narayan and T. Y. Tan (North Holland-New York) 2 (1981) 173.

[5] Stein, H. J., KnAPP, J. A. and PeERCY, P. S., Laser and Electron Beams interaction with solids, edited by B. R. Appleton and G. K. Celler, Elsevier-Amsterdam (1983) 385.

[6] Luo, J., McMarr, P. J. and Vedam, K., Defects in semiconductors, edited by S. Mahajan and J. W. Corbett (North Holland-New York) 14 (1983) 529.

[7] Johnson, N. M., Regolini, J. L. and BARTElinK, D. J., Appl. Phys. Lett. 36 (1980) 425.

[8] Jellison, G. E., Cleland, J. W. and Yang, R. T., Defects in semiconductors, edited by J. Narayan and T. Y. Tan (North Holland-New York) 2 (1981) 241.

[9] Kimerling, L. C. and Benton, J. L., Laser and Electron Beam Processing of Materials, edited by C. W. White and P. S. Peercy (Academic PressNew York) (1980) 385.
[10] Perloff, D. S., Solid Stat. Electron. 20 (1977) 681.

[11] Van Der Pauw, L. J., Philips Res. Rep. 13 (1958) 1.

[12] Csepregi, L., Kennedy, E. F., Gallagher, T. J. and Mayer, J. W., J. Appl. Phys. 48 (1973) 4234.

[13] Nelson, R. S., Proc. Europ. Conf. on Ion Implantation (Stevenage, Herts, England) 1970, p. 212.

[14] Fuller, C. S., Defect interactions in semiconductors, chapter 5 of Semiconductors edited by N.B. Hannay (Reinhaold) 1959.

[15] Kokorowski, S., Olson, G. and Hess, L., J. Appl. Phys. 53 (1982) 921.

[16] Boltaks, B., Diffusion et défauts ponctuels dans les semiconducteurs (Edition Mir, Moscou) 1977, p. 186.

[17] KIRÈEV, P., La physique des semiconducteurs (Edition Mir, Moscou) 1975.

[18] Morin, F. J. and Maita, J. P., Phys. Rev. 96 (1954) 28.

[19] MotT, N. F. and Davis, E. A., Electronic Processes in Non-Crystalline Materials (Clarendon, Oxford) 1975, p. 199.

[20] Friedman, L., J. Non-Crystall. Solids 6 (1971) 329.

[21] Sclar, N., Phys. Rev. 104 (1956) 1548. 